\title{
Hepatic portal venous gas in a patient undergoing chemotherapy for non-small cell lung cancer
}

\author{
Akihiro Sakamoto $\cdot$ Nobuhiro Matsumoto $\cdot$ Yasuji Arimura • \\ Shigehisa Yanagi $\cdot$ Hirotoshi Iiboshi $\cdot$ Masatoshi Tokojima • \\ Shu-ichi Yamashita $\cdot$ Masamitsu Nakazato
}

Received: 14 May 2012/ Accepted: 21 August 2012/Published online: 29 September 2012

(C) The Japan Society of Clinical Oncology 2012

\begin{abstract}
A 71-year-old man was diagnosed with c-stage IV non-small cell lung cancer and treated with anticancer chemotherapy. On the 29th day of chemotherapy, the patient had slight upper abdominal fullness and pain. Ultrasonography showed gas flow in the portal vein and computed tomography confirmed the presence of gas in the peripheral lesion of the liver. We made a diagnosis of hepatic portal venous gas. He was successfully treated with conservative management. No mechanical or bacterial factors were identified, and the occurrence of HPVG in our case was considered to be related to his cancer-bearing condition and the chemotherapy agents. Although HPVG during chemotherapy is extremely rare, we should be aware of this complication in patients undergoing chemotherapy.
\end{abstract}

Keywords Hepatic portal venous gas - Non-small cell lung cancer $\cdot$ Anticancer chemotherapy

\section{Introduction}

Ever since hepatic portal venous gas (HPVG) was first reported by Wolfe and Evans in 1955 [1], it has been associated with a grave condition and poor prognosis. Although the mortality rate from HPVG decreased from $75 \%$ in 1978 [2] to $39 \%$ in 2001 [3], it is still a lifethreatening and ominous condition. Despite the earlier

A. Sakamoto $(\bowtie) \cdot$ N. Matsumoto $\cdot$ Y. Arimura $\cdot$ S. Yanagi .

H. Iiboshi · M. Tokojima - S. Yamashita $\cdot$ M. Nakazato

Neurology, Respirology, Endocrinology, and Metabolism,

Department of Internal Medicine, Faculty of Medicine,

University of Miyazaki, 5200, Kihara, Kiyotake,

Miyazaki 889-1692, Japan

e-mail: as-nz@hotmail.co.jp studies' recommendation of immediate surgical intervention, there have been some reports of the noninvasive management of HPVG. Indeed, in the case described in the present report, we also managed our patient conservatively. Various diseases have been considered to be causes of the condition, including bowel ischemia, infectious colitis, intestinal obstruction, intra-abdominal abscess, and others [3-5]. Although there have been only a couple of case reports of HPVG related to chemotherapy so far, our case highlights the likelihood of developing HPVG during chemotherapy.

\section{Case report}

A 71-year-old man with a nodular opacity in his left lung apex on a chest X-ray was admitted to our hospital. Transbronchial lung biopsy led to the pathological diagnosis of large cell neuroendocrine carcinoma. Bone metastases were detected in his left scapula and left iliac bone. There were no other findings of metastases on imaging tests. We made the diagnosis of c-stage IV nonsmall cell lung cancer. Zoledronic acid and a time-released formulation of morphine sulfate were initiated to treat the bone metastases and the pain associated with the bone lesions. Anticancer chemotherapy consisting of carboplatin and paclitaxel was administered. On the 29th day of chemotherapy, the patient complained of abdominal fullness and slight pain in the upper abdomen without signs of peritoneal irritability. He had no past history related to his abdominal symptoms. An examination of his blood chemistry showed an elevation of alkaline phosphatase (ALP) and $\gamma$-guanosine triphosphate $(\gamma$-GTP). White blood cells, C-reactive protein, and bilirubin were within normal limits. Ultrasonographic examination demonstrated hyperechoic 


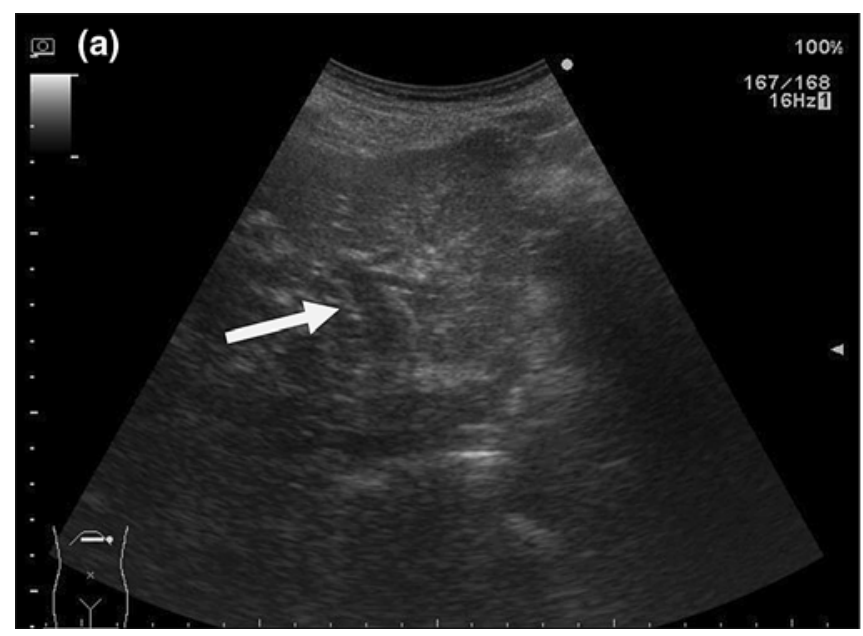

Fig. 1 Ultrasonography and abdominal CT on the day of HPVG onset. a Ultrasonography shows hyperechoic particles flowing in the portal vein only a few hours after the patient complained of

fine particles in the portal vein in real time (Fig. 1a). A subsequent abdominal CT showed gas in the peripheral part of the liver, within $2 \mathrm{~cm}$ from the liver capsule (Fig. 1b). There was no evidence of ischemic bowel disease or pneumatosis intestinalis. We made the diagnosis of HPVG.

The patient was afebrile and hemodynamically stable. Although he had mild abdominal tenderness, no peritoneal signs were presented. He also complained of a strong desire to defecate, and his abdominal symptoms were markedly improved after defecation. We decided to treat him conservatively without surgical intervention. He was placed under strict prohibition of oral intake of any food or liquid, and a nasogastric tube was placed to decompress the digestive system. Prophylactic broad-spectrum antibiotic was administered. His abdominal pain had improved almost completely the next day, and he remained stable afterward. Follow-up ultrasonography and abdominal CT at 2 and 12 days, respectively, after the onset of HPVG showed complete disappearance of the gas in the liver (Fig. 2). ALP and $\gamma$-GTP became normal at six days after the onset of HPVG.

The patient's performance status was good enough for him to continue to undergo chemotherapy. Considering the likely association with HPVG, chemotherapy with carboplatin and paclitaxel was discontinued. Second-line therapy with pemetrexed was administered with careful management of constipation. A recurrence of HPVG was not found after that.

\section{Discussion}

Ever since the first case was reported in 1955 [1], HPVG has been considered to be a rare and typically ominous

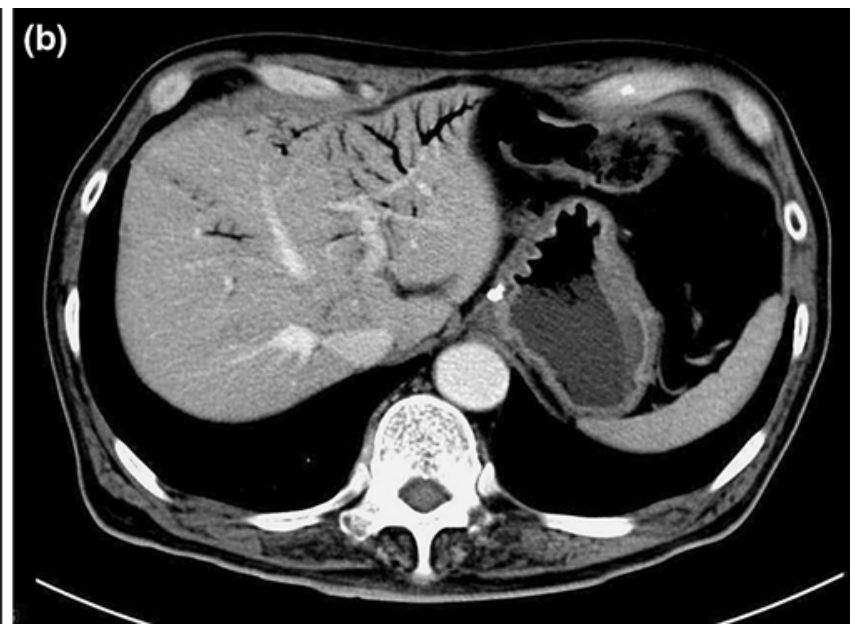

abdominal symptoms. b Adominal CT shows the presence of gas in the peripheral part of the liver, within $2 \mathrm{~cm}$ of the liver capsule

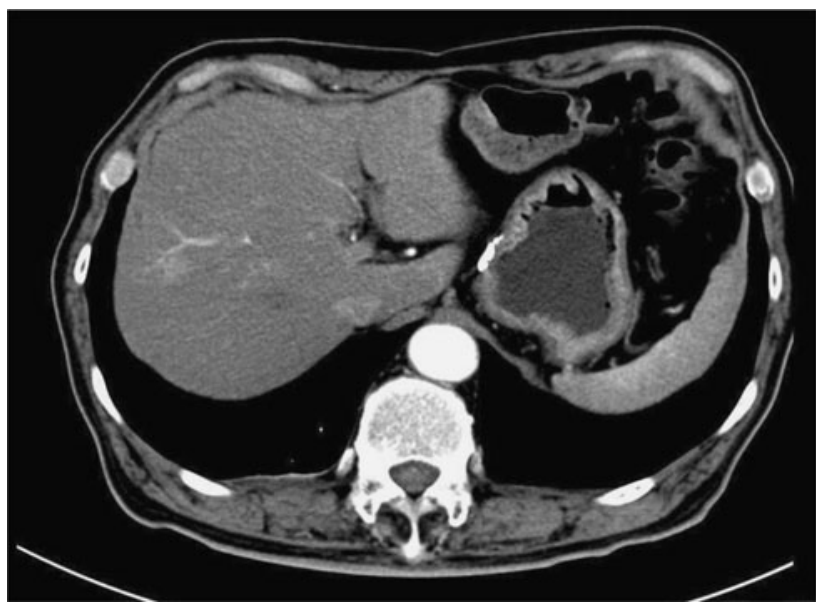

Fig. 2 Follow-up abdominal CT on the 12th day after the onset of HPVG. Complete disappearance of gas in the portal venous system is shown

finding. To our knowledge, the occurrence of HPVG in a patient during chemotherapy is extremely rare; only a few cases have been reported so far, and the causes were not well confirmed.

As for the development of HPVG, three major mechanisms of gas production have been postulated [4-6]: (1) damage to the gastrointestinal mucosa, such as bowel ischemia; (2) bowel distension with increased intraluminal pressure, such as bowel obstruction, ileus, etc.; (3) bacteremia involving the portal venous system with the production of gas. In our case, the rapid improvement of symptoms after defecation, the absence of any finding of severe inflammation, and the negative blood cultures suggest that any kind of bacteremia can be ruled out. In addition, no causes of HPVG (including metastatic sites) were detected with the colonoscopy that was performed 
afterward. There are a couple of reports of HPVG as a complication of anticancer chemotherapy [7-9]. Although the authors failed to elucidate the mechanism of HPVG in their case, the damage to intestinal mucosa and increased mucosal permeability associated with chemotherapy agents cannot be completely ruled out as the cause in our case. Despite the high incidence of toxicity, severe gastrointestinal complications due to carboplatin and paclitaxel are comparatively rare. However, since the intestinal mucosa is highly proliferative, the possibility that these drugs might have caused intestinal mucosal damage cannot be excluded. Furthermore, since a long-acting opiate was initiated for the pain associated with bone metastases, the patient naturally had severe constipation as a side effect. The patient's decreased physical activity and the gastrointestinal symptoms related to chemotherapy such as nausea or appetite loss could have had some effects on his constipation. No cases of HPVG caused by mere constipation have been reported. However, considering the rapid improvement of his symptoms after defecation, we suppose that, aside from the effects of chemotherapy agents, an increased intraluminal pressure due to ileus-like severe constipation played an important role in his development of HPVG.

The management of HPVG has changed over the past several decades. Previously, most cases of HPVG were thought to be associated with intestinal ischemia that led to extended bowel necrosis and fatal outcomes, and it was thought that exploratory surgery was imperative as soon as possible to detect and treat the causative diseases. Although sensitive medical modalities make it possible to identify HPVG in its early stages, as in our case, HPVG associated with bowel necrosis has a poor prognosis even today, with a mortality rate of $75 \%$ [3]. However, HPVG with nonischemic conditions such as ulcerative colitis, Crohn's disease, intestinal obstruction, or complications of endoscopic procedures have more favorable outcomes, and can be treated without surgery. In our case, because of the benign nature of the causative condition, our patient improved with only conservative management, without surgical intervention. The cause of the elevation of his ALP and $\gamma$-GTP was not obvious because of the lack of any apparent pathological condition in the biliary system. Since a simple elevation of the portal venous pressure usually does not elevate these enzymes, we suppose that the decreased blood flow in the portal venous system due to the inflow of gas could be the cause.

In conclusion, we speculate that chemotherapy agents, severe constipation caused by the effect of opiate analgesic, and the decreased physical activity (an adverse effect of chemotherapy) worked cooperatively as the causes of HPVG in the present case. Constipation was considered to have played an especially important role in the development of HPVG in our case. It is therefore important to be aware that HPVG could occur during chemotherapy as a complication of chemotherapy agents and severe constipation related to a cancer therapy.

Conflict of interest The authors declare that they have no conflict of interest.

\section{References}

1. Wolfe J, Evance W (1955) Gas in the portal veins of the liver in infants; a roentgenographic demonstration with postmortem anatomical correlation. Am J Roentgenol Radium Ther Nucl Med $74: 486-488$

2. Liebman P, Patten M, Manny J et al (1978) Hepatic-portal venous gas in adults: etiology, pathophysiology and clinical significance. Ann Surg 187:281-287

3. Kinoshita H, Shinozaki M, Tanimura H et al (2001) Clinical features and management of hepatic portal venous gas: four case reports and cumulative review of the literature. Arch Surg 136:1410-1414

4. Alqahtani S, Coffin C, Burak K et al (2007) Hepatic portal venous gas: a report of two cases and a review of the epidemiology, pathogenesis, diagnosis and approach to management. Can J Gastroenterol 21:309-313

5. Peloponissios N, Halkic N, Pugnale M et al (2003) Hepatic portal gas in adults: review of the literature and presentation of a consecutive series of 11 cases. Arch Surg 138:1367-1370

6. Chang C, Shun H, Chuang C (2009) Hepatic portal venous gas induced by emphysematous pyelonephritis: a rare case in hemodialytic women. Am J Emerg Med 27:e1171-e1173

7. Kung D, Ruan D, Chan R et al (2008) Pneumatosis intestinalis and portal venous gas without bowel ischemia in a patient treated with irinotecan and cisplatin. Dig Dis Sci 53:217-219

8. Ortega J, Hayes J, Antonia S (2009) Hepatic portal venous gas in a patient with metastatic non-small cell lung cancer on bevacizumab therapy: a case report and review of the literature. Cancer Chemother Pharmacol 65:187-190

9. Zalinski S, Scatton O, Jacqmin S et al (2009) Portal venous gas following chemotherapy for colorectal cancer liver metastasis. Eur J Surg Oncol 35:557-560 